\title{
Preoperative CT imaging as an accurate diagnostic modality for negative laparotomy for patients with acute right lower abdominal pain
}

\author{
Taher H Elwan, ${ }^{a} M D$; Mokhtar Abdelrhman Bahbah, ${ }^{a} M D$; \\ Ashraf M Abd elkader, ${ }^{a} M D$; Islam M El shazly, ${ }^{b} M D$
}

\begin{abstract}
a) Department of General Surgery, Benha University, Benha, Egypt. b) Department of Radiology, Benha University, Benha, Egypt.
\end{abstract}

\begin{abstract}
Objectives: Evaluation of the diagnostic yield of preoperative abdominal CT imaging of patients presenting by acute right lower abdominal pain and the probability for reduction of negative appendectomy rates in these patient.

Patients \& methods: The present study aimed to include patients presenting with acute right lower abdominal pain who were admitted as acute abdominal emergency under observation. All enrolled patients underwent clinical examination and laboratory investigations to provide a probable clinical diagnosis. All patients underwent abdominal ultrasonography (US) and then scanning with multi-detector row CT. All patients with clinical suspicion for need of surgical exploration underwent laparotomy and excised specimens were examined pathologically. Patients who became stable with negative US and/or CT were maintained under-observation till 24 hours and were discharged.

Results: One hundred and seventeen patients underwent surgical exploration including 81 patients who had emergency surgery and 36 who had surgery on elective basis during observation period. Pathological examination confirmed positive diagnosis of acute abdomen in 78 patients and 39 patients were pathologically free (Negative laparotomy). Clinical diagnosis defined 79, abdominal US defined 83 and CT defined 74 patients as having positive acute abdominal condition. Preoperative CT showed a significantly higher test validity characters in comparison to abdominal US and clinical examination with sensitivity rate of $94.6 \%$, specificity rate of $90.7 \%$ and accuracy rate for diagnosis of $93.2 \%$. Statistical analysis defined preoperative CT as the best predictor for negative laparotomy.

Conclusion: Preoperative CT for patients with acute right lower abdominal pain reduces the negative laparotomy rate, improves true positive surgical rate and is mandatory especially in suspicious cases. Moreover, preoperative CT could help differential diagnosis of the underlying pathology and so can modify surgical decision.
\end{abstract}

Key words: Preoperative, CT, acute right lower abdominal pain, negative laparotomy.

\section{Introduction:}

Despite being an ancient disease that dates since early history, diagnosis and proposed lines of management for right lower abdominal pain which is mostly due to appendicitis is still a matter of research. The achievement of proper diagnosis irrespective of the cost of methods used could reduce the consumption of resources through reduction of the rate of negative surgery with its consequences as reduction of days off work and allowing direction of health resources towards the ideal target. ${ }^{1-3}$

The diagnosis of acute appendicitis is not always clear clinically since the specificities of the classic clinical symptoms (periumbilical pain migrating to the right lower quadrant, nausea, and anorexia) range from $37 \%$ to $53 \%$. If acute appendicitis is diagnosed as simple 
appendicitis, the recovery time is relatively short time without any complications. However, consequences of missing appendicitis are severe, in perforated or gangrenous appendicitis due to the delay of operation, the hospital stay, the cost, and the incidence of early, as well as delayed complications are increased drastically in terms of morbidity and mortality. 4,5

Improvement of radiological evalution and evolution of new inflammatory markers allowed higher incidence of early diagnosis and treatment for acute appendicitis, but the incidence of early detection for complicated and/or malpresented appendicitis is still limited. 3,6-8

On the other hand, historically, negative appendectomy rates of $20 \%-25 \%$ and as high as $40 \%$ in women have been considered acceptable. The magnitude of the problem of negative appendectomy is much higher in special situations as abnormal presentation, abnormal site of pain or site of pain referral and in children. Women showed generally higher negative appendectomy rates due to gynecologic disease, which can confound the diagnosis of appendicitis. Also, pregnancy is an important cause for high negative laparotomy for acute abdomen.9-12

The current study aimed to evaluate the diagnostic yield of preoperative abdominal CT imaging of patients presenting by acute right lower abdominal pain and the probability for reduction of negative appendectomy rates in such patient population.

\section{Patients and methods:}

The present prospective study was conducted at General Surgery Department, Benha University Hospital since May 2010 till May 2012. After approval of the study protocol by the Local Ethical Committee and obtaining written fully informed patients' or nearest relative consent, the study included 117 patients presenting to Emergency Department with acute right lower abdominal quadrant pain and were admitted as acute abdominal emergency under observation.

All enrolled patients were clinically examined for demographic and constitutional data. History of recurrent pain, nausea and/or vomiting, fever, constipation or diarrhea was undertaken. Clinical data including tenderness, rebound tenderness and special clinical signs were determined. Blood samples were taken for complete blood counting. Probable clinical diagnosis was determined depending on clinical and laboratory data.

All patients underwent abdominal ultrasonography and then scanning with multidetector row CT (HiSpeed Advantage or LightSpeed; GE Medical Systems, Milwaukee, Wis) with 1.25 - or $5-\mathrm{mm}$ section thickness. Scans obtained at $1.25-\mathrm{mm}$ collimation were reconstructed to $5-\mathrm{mm}$ section thickness. Intravenous contrast material (iohexol, Omnipaque 350; Nycomed Amersham, Princeton, NJ) $150 \mathrm{ml}$ was injected at a rate of $3-5 \mathrm{ml} / \mathrm{sec}$.

All patients had clinical suspicion for need of surgical exploration were managed emergently after adjustment of general condition especially if there is fever, nausea, vomiting or dehydration. Otherwise, patients who became stable with negative US and/or CT were maintained under-observation till 24 hours and were discharged. Excised specimens were examined pathologically as a gold standard for comparison of diagnostic accuracy.

\section{Statistical analysis:}

Obtained data were presented as mean $\pm \mathrm{SD}$, ranges, numbers and ratios. Results were analyzed using paired Z-test and Chi-square test. Sensitivity \& specificity of diagnostic modalities as predictors for negative laparotomy were evaluated using the receiver operating characteristic (ROC) curve analysis judged by the area under the curve (AUC) and Regression analysis (Stepwise method). Statistical analysis was conducted using the SPSS (Version 15, 2006) for Windows statistical package. P value $<0.05$ was considered statistically significant.

\section{Results:}

The study included 147 patients presented to ED with a picture suggestive of acute abdomen. One hundred and seventeen patients (79.6\%) underwent surgical exploration including 81 patients who had emergency surgery and 36 who had surgery on elective basis during the follow-up period. There were 52 males and 65 females with mean age of 
34.4 7.5; range: $19-45$ years. Patients' demographic, constitutional and clinical data at time of enrollment are shown in Table(1).

Clinical diagnosis depending on presenting symptoms, objective findings and laboratory data, defined 79 patients $(67.5 \%)$ as having acute abdominal condition mostly acute appendicitis, while the remaining 38 patients $(32.5 \%)$ were kept under observation. Abdominal US defined 83 patients (70.9\%) as having acute abdominal condition, while the remaining 34 patients $(29.1 \%)$ were ultrasonographically free. Abdominal CT defined 74 patients $(63.2 \%)$ as having acute abdominal, while the remaining 43 patients were free. All of the 117 patients underwent surgical exploration and operative findings and pathological examination confirmed positive diagnosis of acute abdomen in 78 patients $(66.7 \%)$ and 39 patients $(33.3 \%)$ were pathologically free (Negative Laparotomy), Table(2).

Considering pathological diagnosis as the gold standard, preoperative CT showed a significantly higher test validity characters in comparison to abdominal US and clinical examination with sensitivity rate of $94.6 \%$, specificity rate of $90.7 \%$ and accuracy rate for diagnosis of $93.2 \%$, Table(3), Figure(1).

Using ROC curve for evaluating the predictability of clinical diagnosis, ultrasonographic diagnosis and CT imaging as predictors for negative diagnosis showed that both US and CT imaging are significant sensitive predictors for negative laparotomy, Figure(2). Moreover, regression analysis defined preoperative $\mathrm{CT}$ as the best predictor for negative laparotomy, Tables(4\&5).

Concerning differential diagnosis of cause of acute abdomen; CT imaging defined 20 cases of gynecological emergencies which were misdiagnosed depending on US examination, Figures(3-5). Moreover, CT imaging defined 3 cases of acute diverticulitis without abscess formation in two, but the $3^{\text {rd }}$ was diverticular abscess Figures(6\&7). One case of cancer caecum, Figure(8) forming mass mimicking appendicular mass on US examination was diagnosed on CT imaging. Three cases had impacted stone lower end right ureter, Figure(9). Thirteen cases had complicated appendicitis, Figure(10), while 38 cases had uncomplicated appendicitis, Figures(11-13).

Table (1): Studied patients data.

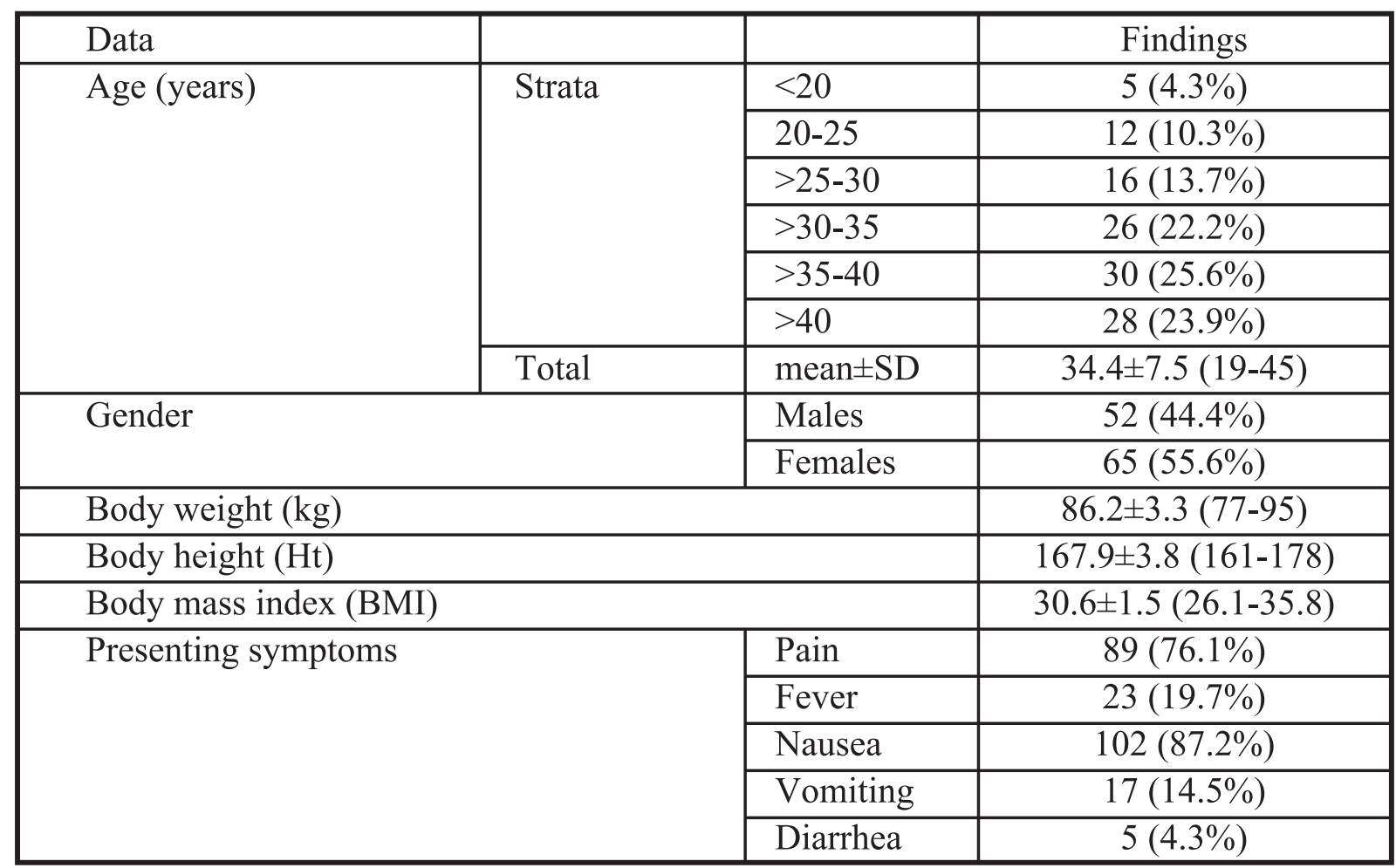

Data are presented as mean $\pm S D$ \& numbers; ranges \& percentages are in parenthesis. 
Table (2): Patients' distribution according to clinical and radiological diagnosis of acute appendicitis compared versus pathological diagnosis.

\begin{tabular}{|c|c|c|c|c|c|}
\hline & & Pathology & Clinical & US & CT \\
\hline Positive & True & 78 & 56 & 61 & 70 \\
\hline & False & 0 & 23 & 22 & 4 \\
\hline Negative & True & 39 & 16 & 24 & 39 \\
\hline & False & & 22 & 10 & 4 \\
\hline
\end{tabular}

Table (3): Test validity characters of clinical and radiological diagnosis of acute appendicitis as the cause of acute abdomen.

\begin{tabular}{|l|c|c|c|c|c|c|}
\hline & Sensitivity & Specificity & PPV & NPV & Accuracy & Statistical analysis \\
\hline Clinical & $71.8 \%$ & $41 \%$ & $70.9 \%$ & $42.1 \%$ & $61.5 \%$ & $\mathrm{X}^{2}=2.124, \mathrm{p}>0.05$ \\
\hline US & $85.9 \%$ & $52.2 \%$ & $73.5 \%$ & $70.6 \%$ & $72.6 \%$ & $\mathrm{X}^{2}=6.33, \mathrm{p}<0.01$ \\
\hline CT & $94.6 \%$ & $90.7 \%$ & $94.6 \%$ & $90.7 \%$ & $93.2 \%$ & $\mathrm{X}^{2}=15.163, \mathrm{p}<0.001$ \\
\hline
\end{tabular}

PPV: Positive predictive value

NPV: Negative predictive value

Table (4): ROC curve analysis of diagnostic yield of clinical and radiological data for prediction of negative laparotomy.

\begin{tabular}{|l|c|c|c|l|r|}
\hline Parameters & AUC & Std Error & Asymptotic Sig. & \multicolumn{2}{|c|}{$95 \%$ Confidence Interval } \\
\cline { 5 - 6 } & & & & Lower & Upper \\
\hline Clinical & 0.435 & 0.057 & $>0.05$ & 0.322 & 0.548 \\
\hline US & 0.298 & 0.054 & $<0.001$ & 0.193 & 0.403 \\
\hline CT & 0.156 & 0.044 & $<0.001$ & 0.071 & 0.241 \\
\hline
\end{tabular}

AUC: area under curve

Std. Error: standard error

Table (5): Regression analysis of diagnostic procedures as the best predictor for prediction of negative laparotomy.

\begin{tabular}{|l|c|c|c|}
\hline & $\beta$ & $\mathrm{T}$ & Sig. \\
\hline Clinical diagnosis & 0.072 & 1.023 & $>0.05$ \\
\hline US scanning & 0.062 & 0.319 & $>0.05$ \\
\hline CT imaging & 0.688 & 10.172 & $<0.001$ \\
\hline
\end{tabular}

$\beta$ : standardized coefficient

t: paired t-test

Sig.: significance 


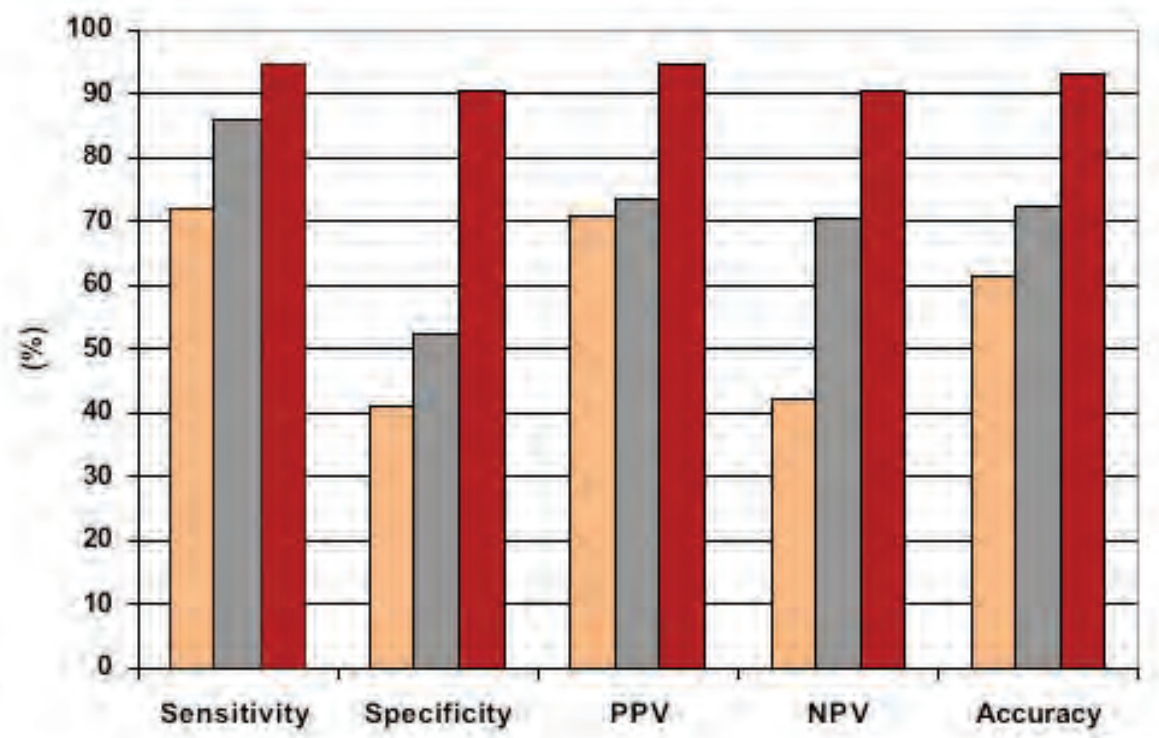

Figure (1): Test vilidity characters of studied diagnostic $\square$ Clinica procedures for diagnosis of pathological acute abdomen.
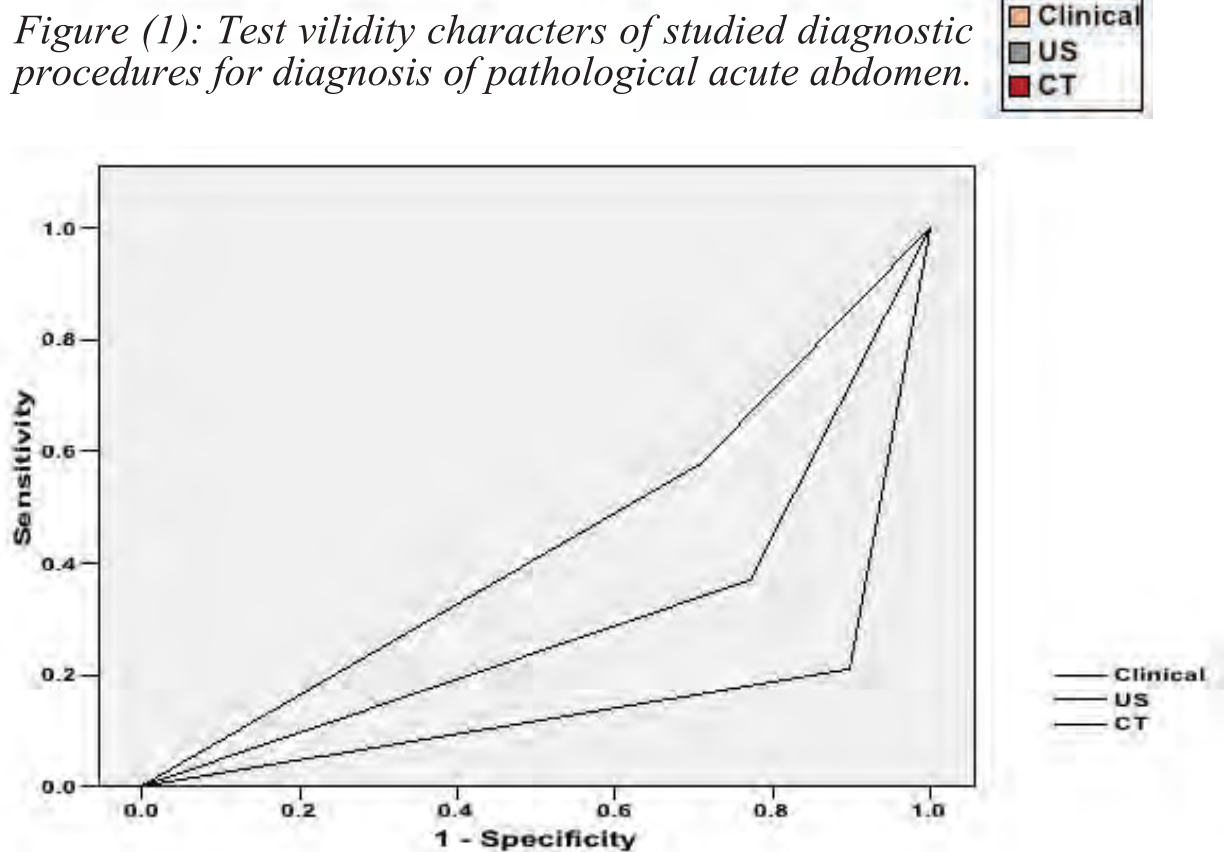

Figure (2): ROC curve analysis for the sensitivity of clinical diagnosis, US scanning and CT imaging for prediction of negative laparotomy.

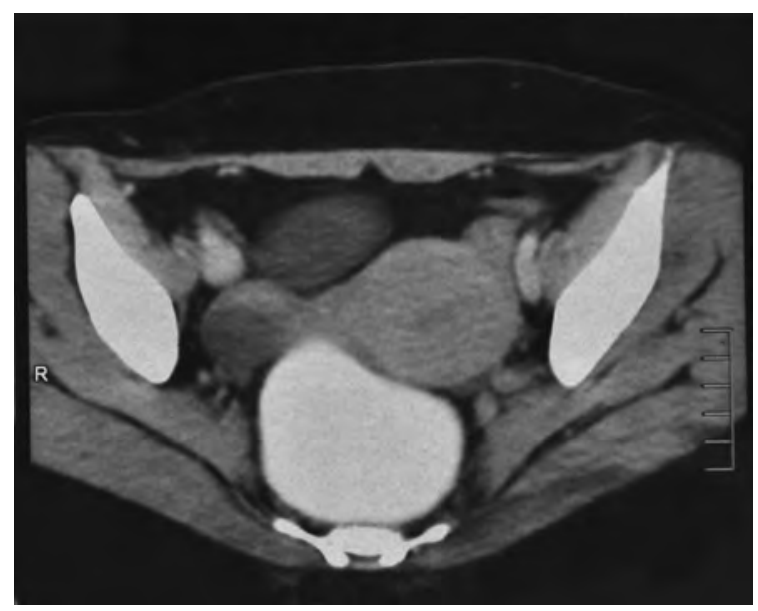

Figure (3): CT image showing complicated right ovarian cyst, operative exploration revealed hemorrhage in a right side ovarian cyst.

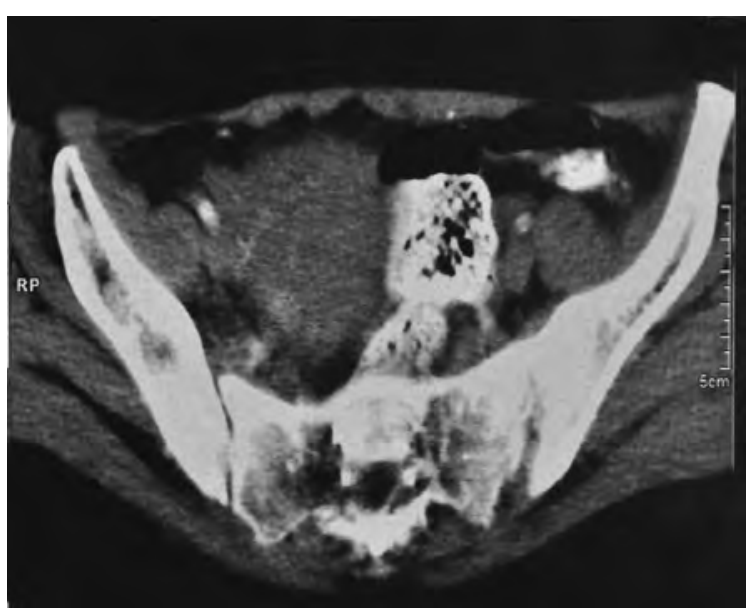

Figure (4): CT image showing right tuboovarian abscess, early stage tubo-ovarian abscess which was drained. 


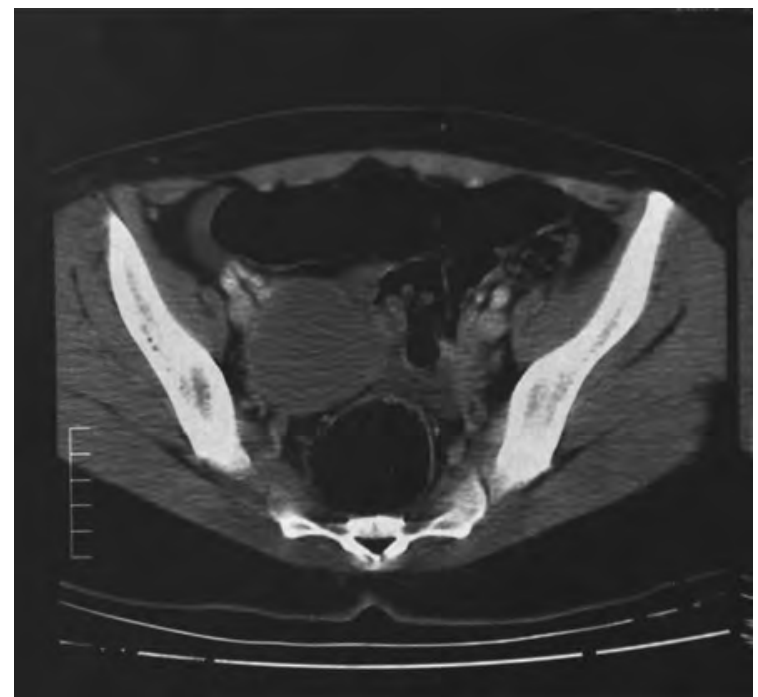

Figure (5): CT image showing complicated ovarian cyst, operative exploration revealed hemorrhage and torsion of right side ovarian cyst.

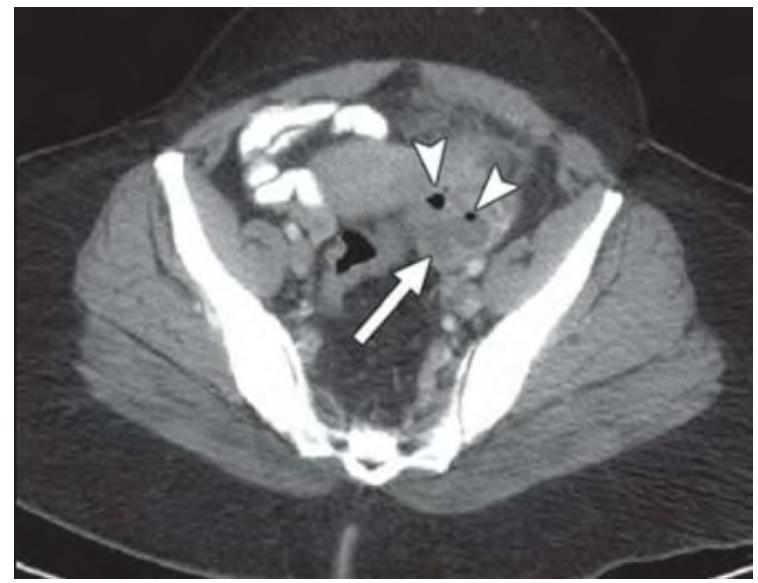

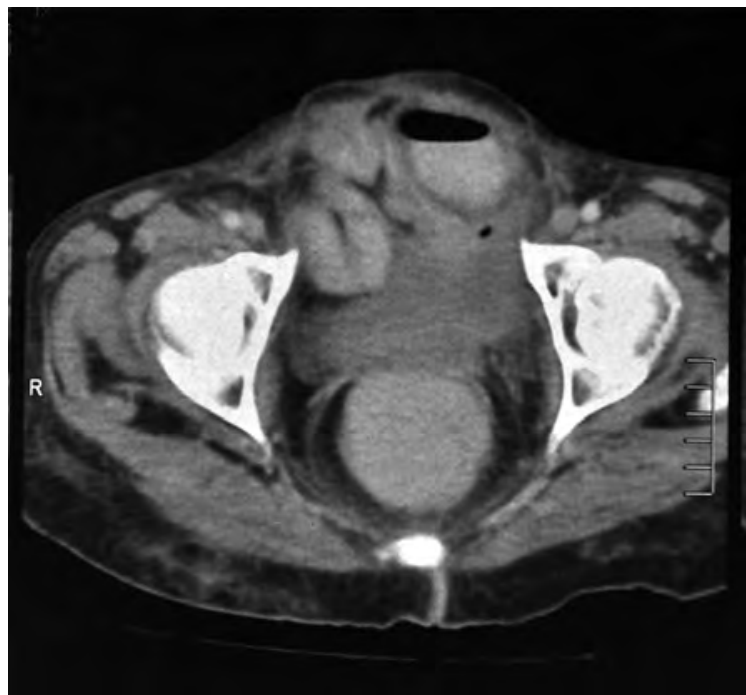

Figure (6): CT image showing pelvic abscess.

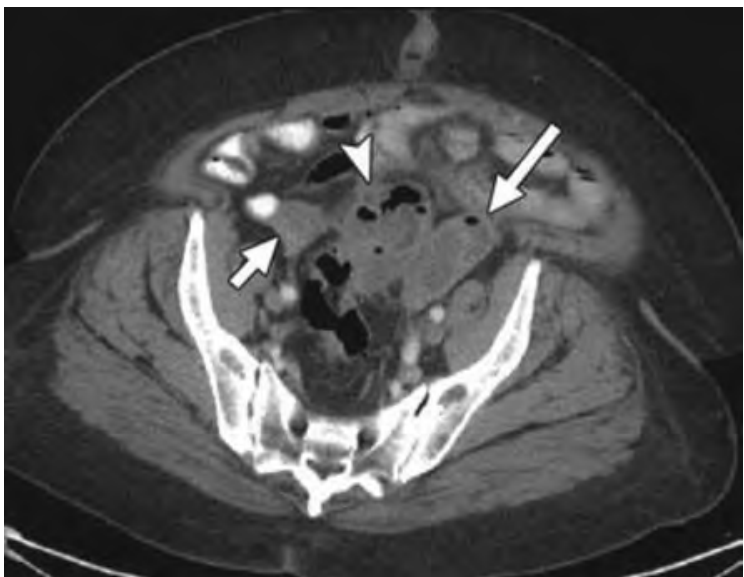

Figure (7a): Shows CT scan of a woman with sigmoid diverticulitis and colosalpingeal fistula. Axial contrast-enhanced CT scan of pelvis shows normal size of right adnexum, enlarged left adnexum containing fluid and foci of gas.

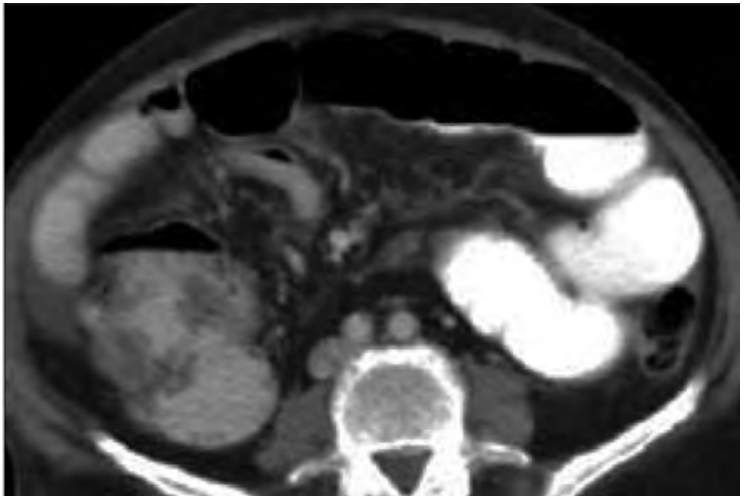

Figure (8): CT image shows asymmetrical mural thickening of the cecum in a case of cecal cancer, resembling an inflammatory mass.

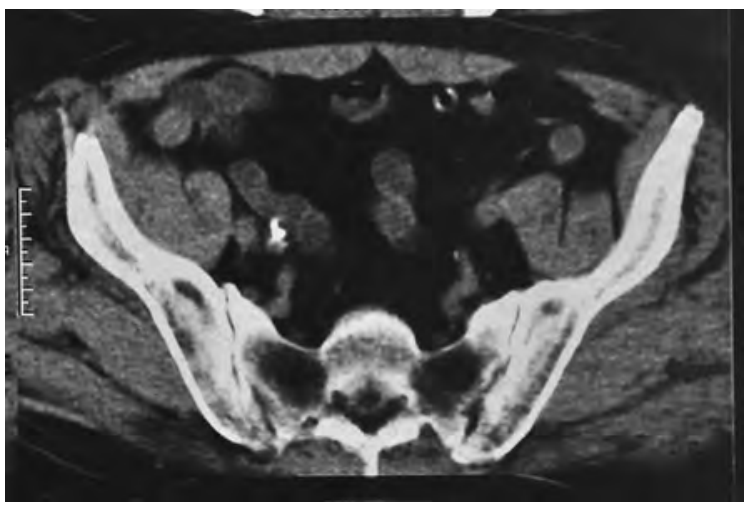

Figure (9): CT image showing impacted stone lower end right ureter. 


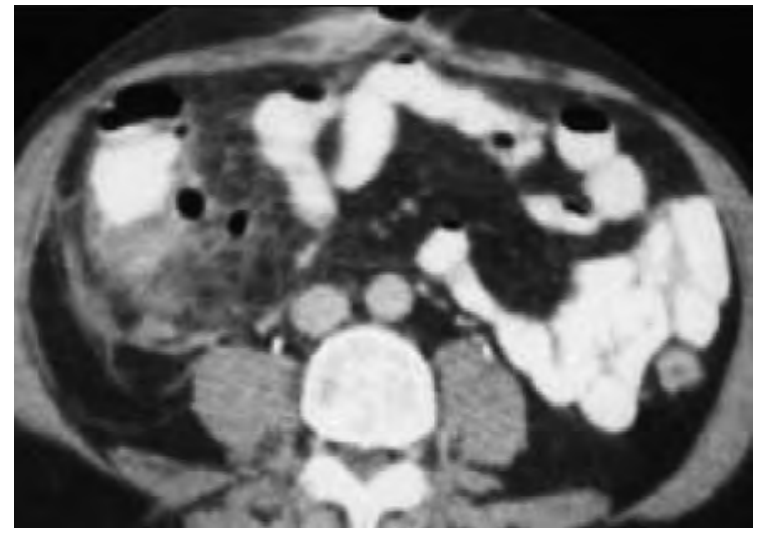

Figure (10): CT image showing an area of ill-defined and variable enhancement with pockets of extraluminal gas is present due to an appendiceal abscess. Note that there is thickening of the peri-cecal fascia.

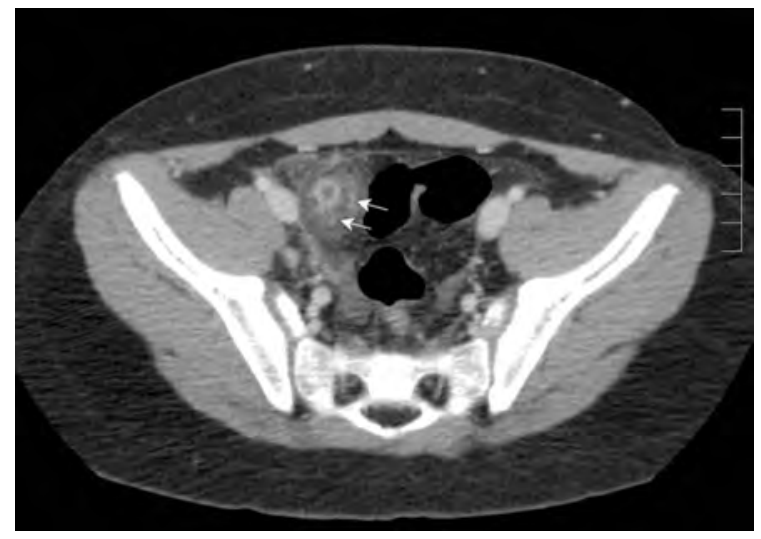

Figure (12): CT showing enlarged appendix with thickened wall and dilated lumen and appendiceal wall enhancement; a picture indicting non-complicated appendicitis.

\section{Discussion:}

Reduction of negative appendectomy rate was evident on application of preoperative CT where the frequency of false positive diagnosis of appendicitis was reduced from about 19\% depending on clinical judgment and/or abdominal US to $3.4 \%$ and the frequency of true negative diagnosis of appendicitis was raised from about $14 \%$ and $19 \%$ depending on clinical judgment or abdominal US, respectively to $33.3 \%$, thus reliance on preoperative $\mathrm{CT}$ could spare surgery in about one-third of examined patients with accuracy of diagnosis of $93.2 \%$.

Considering pathological diagnosis as the gold standard, preoperative CT showed a significantly higher test validity characters in comparison to abdominal US and clinical

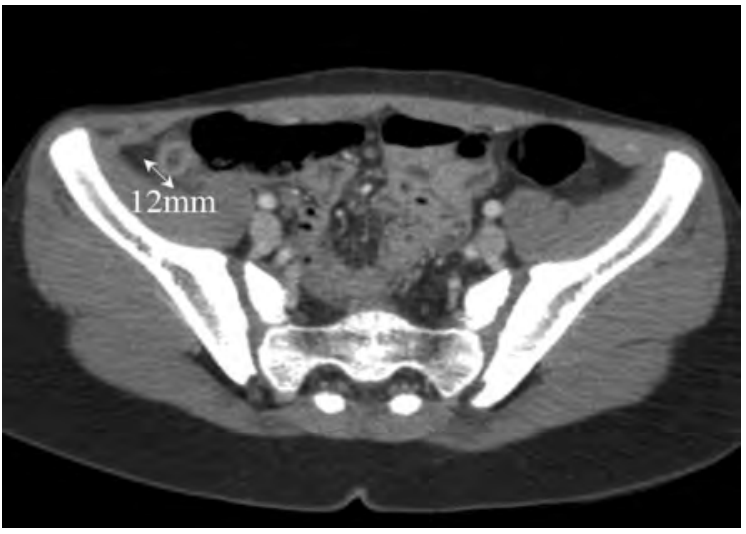

Figure (11): CT showing enlarged appendix with thickened wall and dilated lumen; a picture indicting non-complicated appendicitis.

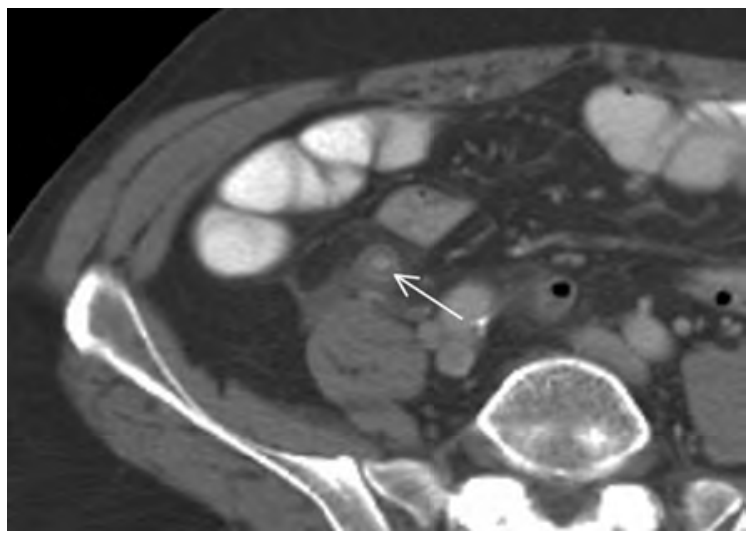

Figure (13): CT showing enlarged appendix with thickened wall and dilated lumen and periappendiceal fat stnding; a picture indicting non-complicated appendicitis.

examination with sensitivity rate of $94.6 \%$, specificity rate of $90.7 \%$ and accuracy rate for diagnosis of $93.2 \%$. Moreover, regression analysis defined preoperative $\mathrm{CT}$ as the best predictor for pathological acute abdominal pain, despite the significantly wider area under curve for abdominal US as detected by ROC curve analysis.

These data and reported figures go in hand with Krajewski et al. ${ }^{13}$ who reported that the negative appendectomy rate was $8.7 \%$ when using CT compared with $16.7 \%$ when using clinical evaluation alone with significantly lower negative appendectomy rate during the $\mathrm{CT}$ era compared with the pre-CT era and concluded that routine $\mathrm{CT}$ in all patients presenting with suspected appendicitis could reduce the rate of unnecessary surgery without 
increasing morbidity.

Rosen et al.14 documented that among adult patients presenting with clinical signs of acute appendicitis, the sensitivity and specificity of CT are greater than those of ultrasound, with improved performance when CT is performed with intravenous contrast. Poletti et al. ${ }^{15}$ evaluated an algorithm integrating ultrasound and low-dose unenhanced CT with oral contrast medium in the assessment of acute appendicitis, to reduce the need of conventional $\mathrm{CT}$ and documented that the proposed algorithm achieved high sensitivity and specificity for detection of acute appendicitis, while reducing the need for standard CT and thus limiting exposition to radiation and to intravenous contrast media.

Petroianu ${ }^{16}$ documented that the advances in imaginology tend to diminish the false positive or negative diagnosis. In experienced hands, ultrasound may have a sensitivity of $90 \%$ and specificity higher than $90 \%$. Helical $\mathrm{CT}$ has reported a sensitivity that may reach 95\% and specificity higher than 95\%. Despite all medical advances, the diagnosis of acute appendicitis continues to be a medical challenge.

In support of the accuracy of abdominal CT for improving outcome of cases of acute abdominal pain; Talanow et al. ${ }^{17}$ described a case with left sided flank pain, workup for nephrolithiasis was negative for renal stones or hydronephrosis, after discharge, the patient presented one week later in the ED with right lower quadrant pain and contrast enhanced CT of the abdomen revealed perforated appendicitis. Abo et al. ${ }^{18}$ tried to determine the relationship between body mass index (BMI) and accuracy of US and CT scan for suspected appendicitis and reported that the sensitivity and specificity of CT for appendicitis are excellent regardless of BMI, while there is a trend of decreasing sensitivity with increasing BMI when using US. Tawk et al. ${ }^{19}$ described a case presenting with a left upper quadrant pain, abdominal U/S was nonconclusive and the diagnosis of acute appendicitis was a long shot, however, on persistence of pain and increasing inflammatory parameters in blood exams a CT scan revealed intestinal mal-rotation with acute appendicitis.
Concerning differential diagnosis of cause of acute abdomen; CT imaging defined 20 cases of gynecological emergencies which were misdiagnosed depending on US examination. Moreover, CT imaging defined 3 cases of acute diverticulitis without abscess formation in two, but the $3^{\text {rd }}$ was diverticular abscess. One case of cancer caecum forming mass mimicking appendicular mass on US examination was diagnosed on CT imaging. These data provide an additional advantage for preoperative CT which may modify the surgical decision. In line with these findings, Purysko et al..$^{20}$ documented that multidetector $\mathrm{CT}$ is an extremely useful noninvasive method for diagnosis and management of not only the most common causes of right lower quadrant abdominal pain such as appendicitis but also less common conditions including inflammatory and infectious conditions involving the ileocecal region; diverticulitis; malignancies; conditions affecting the epiploic appendages, omentum, and mesentery; and miscellaneous conditions.

It could be concluded that preoperative CT for patients with acute right lower abdominal pain reduces the negative surgical rate and improves true positive surgical rate and is mandatory especially in suspicious cases. Moreover, preoperative CT could help differential diagnosis of the underlying pathology and so can modify surgical decision. Wider scale studies are mandatory for evaluation of cost-benefit of considering preoperative $\mathrm{CT}$ as a routine diagnostic procedure.

\section{References:}

1- Purkayastha S, Purkayastha S, Paraskevas P: Acute appendicitis: Weighing up risks and benefits of investigations and treatments. BMJ 2006; 333(7569): 652653.

2- Ilves I, Paajanen HE, Herzig KH, Fagerström A, Miettinen PJ: Changing incidence of acute appendicitis and nonspecific abdominal pain between 1987 and 2007 in Finland. World J Surg 2011; 35(4): 731738.

3- Mayor R, Saenz NC, Kanegaye JT: Acute appendicitis presenting with a painful 
inguinal mass: Complication related to patent processus vaginalis and testicular maldescent. Pediatr Emerg Care 2011; 27(5): 414-416.

4- Johansen LS, Thorup JM, Rasmussen L, Hussain ZB, Kehlet H: Prolonged length of stay and many readmissions after appendectomy. Dan Med Bull 2011; 58(7): A4296.

5- Kong VY, Bulajic B, Allorto NL, Handley J, Clarke DL: Acute appendicitis in a developing country. World J Surg 2012; Epub ahead of print.

6- Vaughan-Shaw PG, Rees JR, Bell E, Hamdan M, Platt T: Normal inflammatory markers in appendicitis: Evidence from two independent cohort studies. JRSM Short Rep 2011; 2(5): 43.

7- Nasiri S, Mohebbi F, Sodagari N, Hedayat A: Diagnostic values of ultrasound and the Modified Alvarado Scoring System in acute appendicitis. Int J Emerg Med 2012; 5(1): 26.

8- Mills AM, Huckins DS, Kwok H, Baumann BM, Ruddy RM, Rothman RE, Schrock JW, Lovecchio F, Krief WI, Hexdall A, Caspari R, Cohen B, Lewis RJ: Diagnostic characteristics of S100A8/A9 in a multicenter study of patients with acute right lower quadrant abdominal pain. Acad Emerg Med 2012; 19(1): 48-55.

9- Gianom D, Köstler T, Goti F, Decurtins M: Effect of new technologies on diagnosis and therapy of acute appendicitis. Praxis 2002; 91(7): 275-284.

10-Hou SK, Chern CH, How CK, Kao WF, Chen JD, Wang LM, Huang CI: Diagnosis of appendicitis with left lower quadrant pain. J Chin Med Assoc 2005; 68(12): 599603.

11-Morse BC, Roettger RH, Kalbaugh CA, Blackhurst DW, Hines WB Jr: Abdominal CT scanning in reproductive-age women with right lower quadrant abdominal pain: Does its use reduce negative appendectomy rates and healthcare costs? Am Surg 2007; 73(6): 580-584.

12-Wei PL, Keller JJ, Liang HH, Lin HC: Acute and adverse pregnancy outcomes:
A nationwide population-based study. J Gastrointest Surg 2012; 16(6): 12041211.

13-Krajewski S, Brown J, Phang PT, Raval $\mathrm{M}$, Brown CJ: Impact of computed tomography of the abdomen on clinical outcomes in patients with acute right lower quadrant pain: A meta-analysis. Can J Surg 2011; 54(1): 43-53.

14-Rosen MP, Ding A, Blake MA, Baker ME, Cash BD, Fidler JL, Grant TH, Greene FL, Jones B, Katz DS, Lalani T, Miller FH, Small WC, Spottswood S, Sudakoff GS, Tulchinsky M, Warshauer DM, Yee J, Coley BD: ACR Appropriateness Criteria? Right lower quadrant pain--suspected appendicitis. J Am Coll Radiol 2011; 8(11): 749-755.

15-Poletti PA, Platon A, De Perrot T, Sarasin F, Andereggen E, Rutschmann O, DupuisLozeron E, Perneger T, Gervaz P, Becker CD: Acute appendicitis: Prospective evaluation of a diagnostic algorithm integrating ultrasound and low-dose CT to reduce the need of standard CT. Eur Radiol 2011; 21(12): 2558-2566.

16-Petroianu A: Diagnosis of acute appendicitis. Int J Surg 2012; 10(3): 115119.

17-Talanow R: An unusual manifestation of acute appendicitis with left flank pain. $J$ Radiol Case Rep 2008; 2(1): 8-11.

18-Abo A, Shannon M, Taylor G, Bachur R: The influence of body mass index on the accuracy of ultrasound and computed tomography in diagnosing appendicitis in children. Pediatr Emerg Care 2011; 27(8): 731-736.

19-Tawk CM, Zgheib RR, Mehanna S: Unusual case of acute appendicitis with left upper quadrant abdominal pain. Int $J$ Surg Case Rep 2012; 3(8): 399-401.

20-Purysko AS, Remer EM, Filho HM, Bittencourt LK, Lima RV, Racy DJ: Beyond appendicitis: Common and uncommon gastrointestinal causes of right lower quadrant abdominal pain at multidetector CT. Radiographics 2011; 31(4): 927-947. 